\title{
Renormalisation constants of quark bilinears in lattice QCD with four dynamical Wilson quarks
}

\section{ETM Collaboration}

Benoit Blossier $^{a}$, Mariane Brinet ${ }^{b}$, Nuria Carrasco ${ }^{c}$, Petros Dimopoulos $^{d}$, Xining $\mathbf{D u}^{b}$, Roberto Frezzotti ${ }^{d, e}$, Vicent Gimenez ${ }^{c}$, Gregorio Herdoiza ${ }^{f}$, Karl Jansen ${ }^{g}$ Vittorio Lubicz $^{h}$, David Palao ${ }^{* e}$, Elisabetta Pallante ${ }^{i}$, Olivier Pène ${ }^{a}$, Konstantin Petrov ${ }^{a}$, Siebren Reker ${ }^{i}$, Giancarlo Rossi ${ }^{d, e}$, Francesco Sanfilippo $^{a}$, Silvano Simula ${ }^{j}$, Luigi Scorzato ${ }^{k}$, Carsten Urbach ${ }^{l}$

${ }^{a}$ Laboratoire de Physique Théorique, CNRS et Université Paris-Sud XI, Bâtiment 210, 91405 Orsay Cedex, France

${ }^{b}$ Laboratoire de Physique Subatomique et de Cosmologie, CNRS/IN2P3/UJF, 53 avenue des Martyrs, 38026 Grenoble, France

${ }^{c}$ Dep. de Fisica Teórica and IFC, Universitat de Valencia-CSIC, Dr. Moliner 50, E-46100 Burjassot, Spain

'Dipartimento di Fisica, Università di Roma "Tor Vergata" Via della Ricerca Scientifica 1, I-00133 Rome, Italy

eINFN Sezione di “Roma Tor Vergata”, Via della Ricerca Scientifica 1, I-00133 Rome, Italy

${ }^{f}$ Dep. de Fisica Teórica and Instituto de Fisica Teórica UAM/CSIC,

Universidad Autonóma de Madrid, E-28049 Cantoblanco, Spain

${ }^{g}$ DESY, Platanenallee 6, D-15738 Zeuthen, Germany

${ }^{h}$ Dipartimento di Fisica, Università Roma Tre and INFN

Via della Vasca Navale 84, I-00146 Rome, Italy

${ }^{i}$ Centre for Theoretical Physics, University of Groningen, Nijenborgh 4, 9747 AG Groningen, The Netherlands

${ }^{j}$ INFN-Roma Tre Via della Vasca Navale 84, I-00146 Rome, Italy

${ }^{k}$ ECT*/FBK - strada delle tabarelle 286 - 38123 - Villazzano (TN) - Italy

${ }^{l}$ HISKP (Theory) and Bethe Center for Theoretical Physics, Rheinische Friedrich-Wilhelms-Universität Bonn, Nussalle 14-16, 53115 Bonn, Germany E-mail: david.palao@roma2.infn.it

We present preliminary results of the non-perturbative computation of the RI-MOM renormalization constants in a mass-independent scheme for the action with Iwasaki glue and four dynamical Wilson quarks employed by ETMC. Our project requires dedicated gauge ensembles with four degenerate sea quark flavours at three lattice spacings and at several values of the standard and twisted quark mass parameters. The RI-MOM renormalization constants are obtained from appropriate $O(a)$ improved estimators extrapolated to the chiral limit.

The XXIX International Symposium on Lattice Field Theory - Lattice 2011

July 10-16, 2011

Squaw Valley, Lake Tahoe, California

\footnotetext{
*Speaker.

${ }^{\dagger}$ Preprint number: DESY 11-235
} 


\section{Introductory remarks and computational setup}

The European Twisted Mass Collaboration (ETMC) has recently performed simulations employing mass degenerate light (up/down) doublet quarks and a heavy mass non-degenerate pair for strange and charm quarks entering in the era of precise and realistic $N_{f}=2+1+1$ dynamical quark lattice computations [1]. In the ETMC setup gluon interactions are described by the Iwasaki action [2]. Fermions are regularised in the maximally twisted mass (Mtm) Wilson lattice formulation $[3,4,5]$. This choice of the fermionic action has the benefit of achieving automatic $\mathrm{O}(a)$-improvement, generally leading to small $\mathrm{O}\left(a^{2}\right)$ lattice artefacts. Up to now ETMC has produced $N_{f}=2+1+1$ dynamical quark gauge configurations at three values of the lattice spacing (namely $a \sim 0.06,0.08$ and $0.09 \mathrm{fm}$ ). Gauge ensembles have been produced at several values of the quark masses with the lowest pseudoscalar mass being of about $270 \mathrm{MeV}$. Obviously the inclusion of the dynamical strange and charm quarks offers the advantage of taking into account a rather important source of systematic effects.

Computation of renormalisation constants (RCs) is a crucial step in order to extract physical quantities from lattice data. It is worth noticing that the Mtm setup offers a rather convenient quark mass renormalisation pattern. Indeed, for the renormalisation of the masses of degenerate and nondegenerate quark pairs one only needs to know the non-singlet $Z_{P}$ and $Z_{S}$ renormalisation constants (and not $Z_{S^{0}}$ ) [4]. RCs of operators with non-zero anomalous dimension need to be computed in the chiral limit and for this reason dedicated lattice simulations employing $N_{f}=4$ light and (for simplicity) degenerate dynamical quarks are required. Indeed we have produced $N_{f}=4$ gauge configuration ensembles corresponding to several sea quark mass values and we determined RCs extrapolating their lattice estimators to the chiral limit. We employed the RI-MOM scheme [6] and the techniques already used for $N_{f}=2$ RCs [7]. However, for the case of $N_{f}=4$ simulations with the action and at the lattice spacing values we are currently using, the implementation of maximal twist (i.e. tuning the PCAC quark mass to zero), which would guarantee $\mathrm{O}(a)$ improvement of RCs, is not a trivial task. In fact in the region of small PCAC quark mass values simulation instabilities occur that lead for it to very large autocorrelation times. Hence we opted for an alternative way, already proposed in Ref. [3], to achieve $\mathrm{O}(a)$ improvement though working out of maximal twist. The method, being based on averaging results obtained at opposite values of the PCAC quark mass, entails the need of doubling the reasonably low CPU time cost for producing gauge simulations at non-zero standard and twisted quark mass. The present contribution is a report of a work in progress. A first presentation and numerical test of our method appeared already in Ref. [8].

\subsection{Computational setup}

We consider the following fermionic lattice action (written in the so called physical basis)

$$
S_{F}^{p h}=a^{4} \sum_{x} \sum_{f=1}^{4} \bar{q}_{f}\left[\gamma \cdot \widetilde{\nabla}-i \gamma_{5} r_{f} e^{i \gamma_{5} r_{f} \theta_{0, f}}\left(-\frac{a}{2} \nabla^{*} \nabla+m_{\mathrm{cr}}\right)+M_{0, f}\right] q_{f}(x)
$$

where $q_{f}$ denotes a singlet quark flavour and $r_{f}$ takes values either -1 or +1 . The chiral quark field 
rotation $\chi_{f} \rightarrow q_{f}=\exp \left[\frac{i}{2}\left(\frac{\pi}{2}-\theta_{0, f}\right) \gamma_{5} r_{f}\right] \chi_{f}$ brings the action into the so-called twisted basis ${ }^{1}$

$$
S_{F}^{t m}=a^{4} \sum_{x} \sum_{f=1}^{4} \bar{\chi}_{f}\left[\gamma \cdot \widetilde{\nabla}-\frac{a}{2} \nabla^{*} \nabla+m_{0, f}+i \gamma_{5} r_{f} \mu_{f}\right] \chi_{f}(x)
$$

The bare mass parameters and the angle $\theta_{0}$ are given by

$$
M_{0, f}=\sqrt{\left(m_{0, f}-m_{\mathrm{cr}}\right)^{2}+\mu_{f}^{2}}, \quad \sin \theta_{0, f}=\frac{m_{0, f}-m_{\mathrm{cr}}}{M_{0, f}}, \quad \cos \theta_{0, f}=\frac{\mu_{f}}{M_{0, f}} .
$$

In practice we make use of $m_{\mathrm{PCAC}}$ to estimate $\left(m_{0, f}-m_{\mathrm{cr}}\right)$. In this way we take as the renormalised quantities the polar quark mass $\hat{M}_{f}=Z_{P}^{-1} M_{f}=Z_{P}^{-1} \sqrt{Z_{A}^{2} m_{\mathrm{PCAC}}^{2}+\mu_{f}^{2}}$ and the angle $\theta_{f}$, complementary to the twisted angle $\omega_{f}\left(\theta_{f}=\pi / 2-\omega_{f}\right)$, given by $\tan \theta_{f}=Z_{A} m_{\mathrm{PCAC}} / \mu_{f}$. As we use four mass degenerate quark flavours and we adopt a partially quenched setup, the knowledge of the four parameters $M_{\text {sea }}, \theta_{\text {sea }}, M_{\text {val }}, \theta_{\text {val }}$ is sufficient to describe our RC computation.

In the following we focus on the evaluation of the RCs of the (non-singlet) quark bilinear operators $^{2} O_{\Gamma}=\bar{\chi}_{f} \Gamma \chi_{f^{\prime}}$ where $\Gamma=S, P, V, A, T$, in the RI' variant of the RI-MOM scheme. One first determines the quark field $\mathrm{RC}, Z_{q}$, through

$$
Z_{q}^{-1} \frac{-i}{12 N(p)} \sum_{\rho}^{\prime}\left[\frac{\operatorname{Tr}\left(\gamma_{\rho} S_{f}(p)^{-1}\right)}{\tilde{p}_{\rho}}\right]_{\tilde{p}^{2}=\mu^{2}}=1, \quad \text { any } f
$$

where $\tilde{p}_{\mu} \equiv \frac{1}{a} \sin a p_{\mu}, \tilde{p}^{2}=\sum_{\mu} \tilde{p}_{\mu}^{2}$ and $S_{f}(p)=a^{4} \sum_{x} e^{-i p x}\left\langle\chi_{f}(x) \bar{\chi}_{f}(0)\right\rangle$ is the Landau gauge quark propagator in momentum space. The sum $\sum_{\rho}{ }^{\prime}$ runs over the Lorentz indices for which $\tilde{p}_{\rho}$ is different from zero and $N(p)=\sum_{\rho}{ }^{\prime} 1$. Then one computes the $\mathrm{RC}, Z_{\Gamma}$, of the operator $O_{\Gamma}$ via

$$
Z_{q}^{-1} Z_{\Gamma}^{\left(f f^{\prime}\right)} \operatorname{Tr}\left[\left(S_{f}^{-1}(\tilde{p}) G_{\Gamma}^{\left(f f^{\prime}\right)}(\tilde{p}, \tilde{p}) S_{f^{\prime}}^{-1}(\tilde{p})\right) P_{\Gamma}\right]_{\tilde{p}^{2}=\mu^{2}}=1, \quad f \neq f^{\prime}
$$

where

$$
G_{\Gamma}^{\left(f f^{\prime}\right)}(p, p)=a^{8} \sum_{x, y} e^{-i p(x-y)}\left\langle\chi_{f}(x)\left(\bar{\chi}_{f} \Gamma \chi_{f^{\prime}}\right)(0) \bar{\chi}_{f^{\prime}}(y)\right\rangle \quad \Gamma=S, P, V, A, T .
$$

with $r_{f^{\prime}}=-r_{f}$ for the Wilson parameters of the (valence) quark flavours $f$ and $f^{\prime}$. We note that RCs are blind to the choice of $\operatorname{sign}\left(r_{f}\right)$ but lattice artefacts in their estimators in general are not.

In our computation we will exploit the fact that the $O\left(a^{2 k+1}\right)$ artefacts occurring in the vacuum expectation values of (multi)local operators $O$ vanish if we take the $\theta$-average defined by $\frac{1}{2}\left[\left.\langle O\rangle\right|_{\hat{M}, \theta}+\left.\langle O\rangle\right|_{\hat{M},-\theta}\right]$. The $\mathrm{O}(a)$ improvement obtained in this way is a consequence of the symmetry $^{3} \mathscr{P} \times\left(\theta_{0} \rightarrow-\theta_{0}\right) \times \mathscr{D}_{d} \times\left(M_{0} \rightarrow-M_{0}\right)$ of the lattice action and occurs for operator expectation values and form factors that are invariant under $\mathscr{P} \times\left(\theta_{0} \rightarrow-\theta_{0}\right)$, see refs. [3, 9]. In particular this holds for our RCs estimators at any value of quark mass, $M_{f}$, and momentum, $\tilde{p}$.

\footnotetext{
${ }^{1}$ For consistency with the standard Wilson fermion notation, the operator RCs are named according to the form the operators take in this basis, where the Wilson term is untwisted.

${ }^{2}$ For the computation of the RCs of the four-fermion operators using the same setup see Ref. [10].

${ }^{3}$ We denote by $\mathscr{P}$ the parity transformation of the fields and by $\mathscr{D}_{d}$ the transformation defined from $\mathscr{D}_{d} q_{f}(x)=$ $e^{3 i \pi / 2} q_{f}(-x), \mathscr{D}_{d} \bar{q}_{f}(x)=e^{3 i \pi / 2} \bar{q}_{f}(-x)$ and $\mathscr{D}_{d} U_{\mu}=U_{\mu}^{\dagger}(-x-a \hat{\mu})$.
} 


\section{Analysis and Results}

We have produced $N_{f}=4$ dynamical quark gauge configurations at three values of the inverse gauge coupling, $\beta=1.90,1.95$ and 2.10, and for each $\beta$ at a number of values of $M^{\text {sea }}$ and (nearly) opposite values of $\theta^{\text {sea }}$ (the $\mathrm{p} / \mathrm{m}$ in the ensemble labels refers to $\operatorname{sign}\left(\theta_{\text {sea }}\right)$ ). An overview of these ensembles and the valence mass parameters chosen for the Landau gauge correlation functions is given in Table 1. Some quark propagator computations are still in progress, as indicated. Hence, the corresponding ensembles can not be used in this analysis.

\begin{tabular}{|c|c|c|c|c|c|c|}
\hline ensemble & $a \mu_{\text {sea }}$ & $a m_{\mathrm{PCAC}}^{\mathrm{sea}}$ & $a M_{0}^{\text {sea }}$ & $\theta^{\text {sea }}$ & $a \mu_{\mathrm{val}}$ & $a m_{\mathrm{PCAC}}^{\mathrm{val}}$ \\
\hline \multicolumn{7}{|c|}{$\beta=1.90$} \\
\hline $4 m$ & 0.0080 & $-0.0390(01)$ & $0.0285(01)$ & $-1.286(01)$ & in prog & $\ldots$ \\
\hline $4 p$ & 0.0080 & $0.0398(01)$ & $0.0290(01)$ & $+1.291(01)$ & in progress & $\ldots$ \\
\hline $3 \mathrm{~m}$ & 0.0080 & $-0.0358(02)$ & $0.0263(01)$ & $-1.262(02)$ & in progress & $\ldots$ \\
\hline $3 p$ & 0.0080 & $+0.0356(02)$ & $0.0262(01)$ & $+1.260(02)$ & in progress & $\ldots$ \\
\hline $2 \mathrm{~m}$ & 0.0080 & $-0.0318(01)$ & $0.0237(01)$ & $-1.226(02)$ & in progress & $\ldots$ \\
\hline $2 p$ & 0.0080 & $+0.0310(02)$ & $0.0231(01)$ & $+1.218(02)$ & in progress & $\ldots$ \\
\hline $1 \mathrm{~m}$ & 0.0080 & $-0.0273(02)$ & $0.0207(01)$ & $-1.174(03)$ & in progress & $\ldots$ \\
\hline $1 \mathrm{p}$ & 0.0080 & $+0.0275(04)$ & $0.0209(01)$ & $+1.177(05)$ & in progress & $\ldots$ \\
\hline \multicolumn{7}{|c|}{$\beta=1.95$} \\
\hline $1 \mathrm{~m}$ & 0.0085 & $-0.0413(02)$ & $0.0329(01)$ & $-1.309(01)$ & {$[0.0085, \ldots, 0.0298]$} & $-0.0216(02)$ \\
\hline $1 \mathrm{p}$ & 0.0085 & $+0.0425(02)$ & $0.0338(01)$ & $+1.317(01)$ & {$[0.0085, \ldots, 0.0298]$} & $+0.0195(02)$ \\
\hline $7 \mathrm{~m}$ & 0.0085 & $-0.0353(01)$ & $0.0285(01)$ & $-1.268(01)$ & {$[0.0085, \ldots, 0.0298]$} & $-0.0180(02)$ \\
\hline $7 p$ & 0.0085 & $+0.0361(01)$ & $0.0285(01)$ & $+1.268(01)$ & {$[0.0085, \ldots, 0.0298]$} & $+0.0181(01)$ \\
\hline $8 \mathrm{~m}$ & 0.0020 & $-0.0363(01)$ & $0.0280(01)$ & $-1.499(01)$ & {$[0.0085, \ldots, 0.0298]$} & $-0.0194(01)$ \\
\hline $8 p$ & 0.0020 & $+0.0363(01)$ & $0.0274(01)$ & $+1.498(01)$ & {$[0.0085, \ldots, 0.0298]$} & $+0.0183(02)$ \\
\hline $3 \mathrm{~m}$ & 0.0180 & $-0.0160(02)$ & $0.0218(01)$ & $-0.601(06)$ & {$[0.0060, \ldots, 0.0298]$} & $-0.0160(02)$ \\
\hline $3 p$ & 0.0180 & $+0.0163(02)$ & $0.0219(01)$ & $+0.610(06)$ & {$[0.0060, \ldots, 0.0298]$} & $+0.0162(02)$ \\
\hline $2 \mathrm{~m}$ & 0.0085 & $-0.0209(02)$ & $0.0182(01)$ & $-1.085(03)$ & {$[0.0085, \ldots, 0.0298]$} & $-0.0213(02)$ \\
\hline $2 p$ & 0.0085 & $+0.0191(02)$ & $0.0170(02)$ & $+1.046(06)$ & {$[0.0085, \ldots, 0.0298]$} & $+0.0191(02)$ \\
\hline $4 \mathrm{~m}$ & 0.0085 & $-0.0146(02)$ & $0.0141(01)$ & $-0.923(04)$ & {$[0.0060, \ldots, 0.0298]$} & $-0.0146(02)$ \\
\hline $4 p$ & 0.0085 & $+0.0151(02)$ & $0.0144(01)$ & $+0.940(07)$ & {$[0.0060, \ldots, 0.0298]$} & $0.0151(02)$ \\
\hline \multicolumn{7}{|c|}{$\beta=2.10$} \\
\hline $5 \mathrm{~m}$ & 0.0078 & $-0.00821(11)$ & $0.0102(01)$ & $-0.700(07)$ & {$[0.0048, \ldots, 0.0293]$} & $-0.0082(01)$ \\
\hline $5 p$ & 0.0078 & $0.00823(08)$ & $0.0102(01)$ & $+0.701(05)$ & {$[0.0048, \ldots, 0.0293]$} & $+0.0082(01)$ \\
\hline $4 m$ & 0.0064 & $-0.00682(13)$ & $0.0084(01)$ & $-0.706(09)$ & in progress & $\ldots$ \\
\hline $4 p$ & 0.0064 & $+0.00685(12)$ & $0.0084(01)$ & $+0.708(09)$ & in progress & $\ldots$ \\
\hline $3 \mathrm{~m}$ & 0.0046 & $-0.00585(08)$ & $0.0066(01)$ & $-0.794(07)$ & {$[0.0025, \ldots, 0.0297]$} & $-0.0059(01)$ \\
\hline $3 p$ & 0.0046 & $+0.00559(14)$ & $0.0064(01)$ & $+0.771(13)$ & {$[0.0025, \ldots, 0.0297]$} & $+0.0056(01)$ \\
\hline $2 \mathrm{am}$ & 0.0030 & $-0.00403(14)$ & $0.0044(01)$ & $-0.821(17)$ & in progress & $\cdots$ \\
\hline $2 a p$ & 0.0030 & $+0.00421(13)$ & $0.0045(01)$ & $+0.843(15)$ & in progress & $\ldots$ \\
\hline
\end{tabular}

Table 1: Overview of produced ensembles at $\beta=1.90,1.95$ and 2.10

The basic ingredient of the calculation, due to Eq.(1.5), is the lattice RC estimator,

$$
Z_{\Gamma}^{\mathrm{Np} / \mathrm{m}} \equiv Z_{\Gamma}^{\mathrm{Np} / \mathrm{m}}\left(M_{0}^{\mathrm{sea}, \mathrm{Np} / \mathrm{m}}, \theta_{0}^{\mathrm{sea}, \mathrm{Np} / \mathrm{m}} ;\left\{M_{j}^{\mathrm{val}, \mathrm{Np} / \mathrm{m}}, \theta_{j}^{\mathrm{val}, \mathrm{Np} / \mathrm{m}}\right\} ; \tilde{p}^{2} ; \beta\right),
$$

where $j$ labels the valence quark polar mass and the momenta $p$ are such that $a^{2} \tilde{p}^{2}$ ranges from 0.5 to 2.5 and that $a^{2} \tilde{p}^{[4]} / \tilde{p}^{2} \leq 0.28$, with $\tilde{p}^{[4]}=\sum_{\rho} \tilde{p}_{\rho}^{4}$. Our analysis goes through the following steps. 


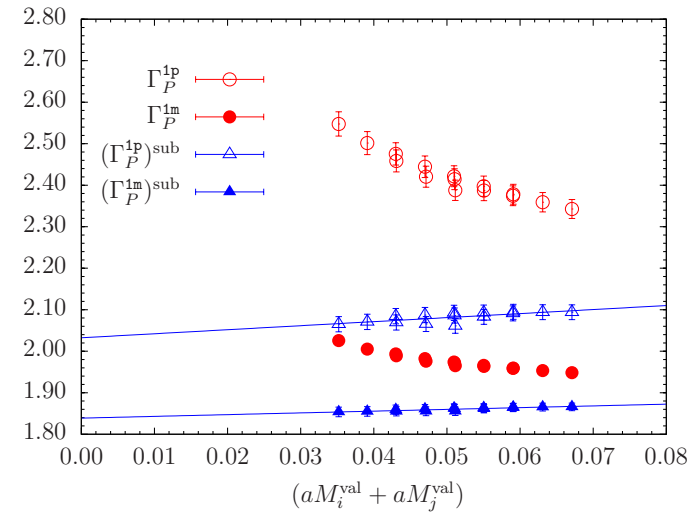

(a)

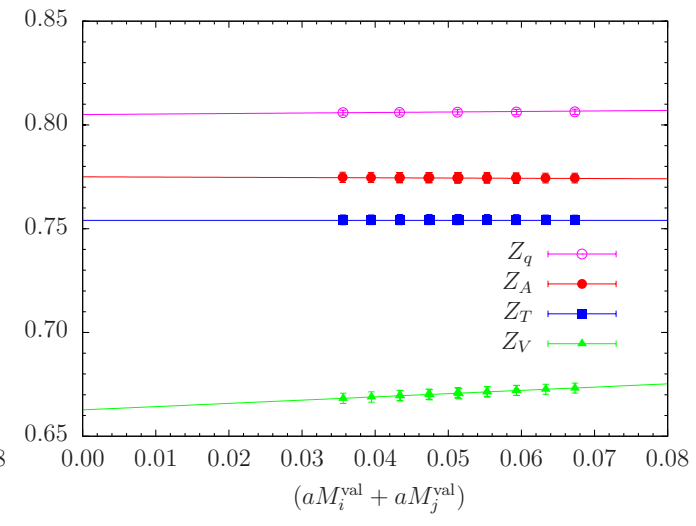

(b)

Figure 1: $\beta=1.95$, ensemble $1 \mathrm{p} / \mathrm{m}$ : (a) Goldstone pole subtraction fit applied separately on $\mathrm{p}$ and $\mathrm{m}$ for estimators of $Z_{P}^{-1}$ at $\tilde{p}^{2} \sim 9.5 \mathrm{GeV}^{2}$; (b) extrapolation of the $\theta$-averaged RC estimators to the chiral limit of $Z_{q}, Z_{A}, Z_{T}$ and $Z_{V}$ at $\tilde{p}^{2} \sim 11.5 \mathrm{GeV}^{2}$. The situation is similar for other ensembles.

(1) Subtract from the RC estimator the $\mathrm{O}\left(a^{2} g^{2}\right)$ cutoff effects at the chiral point, known from Ref. [11] (2) Build the $\mathrm{O}(a)$ improved estimator $Z_{\Gamma}^{\mathbb{N}} \equiv Z_{\Gamma}^{\mathbb{N}}\left(M_{0}^{\text {sea, }, \mathrm{N}}, \theta_{0}^{\text {sea }, \mathrm{N}} ;\left\{M_{j}^{\mathrm{val}, \mathrm{N}}, \theta_{j}^{\mathrm{val}, \mathrm{N}}\right\} ; \tilde{p}^{2} ; \beta\right)=$ $\frac{1}{2}\left[Z_{\Gamma}^{\mathrm{Np}}+Z_{\Gamma}^{\mathrm{Nm}}\right]$.

(3) Extrapolate to the chiral limit value, first in the valence and then in the sea sector.

(4) Evaluate the RCs at a given renormalization scale after taking care of the residual lattice artefacts according to the methods "M1" and "M2" (see Ref. [7] and discussion below).

In step (2) chiral fit Ansätze are inspired to the mass parameter dependence expected from continuum QCD and the Symanzik analysis of lattice artefacts. For the valence chiral extrapolation we considered as fit functions linear combinations of constant, $M_{j}^{\mathrm{val}},\left(M_{j}^{\mathrm{val}}\right)^{2}, M_{j}^{\mathrm{val}} \cos \left(\theta_{j}^{\mathrm{val}}\right)$, and $\left(M_{j}^{\mathrm{val}} \cos \left(\theta_{j}^{\mathrm{val}}\right)\right)^{2}$. For the sea chiral limit linear combinations of $M_{0}^{\text {sea }},\left(M_{0}^{\text {sea }}\right)^{2}$ and $\cos \left(\theta_{j}^{\mathrm{val}}\right)\left(M_{0}^{\text {sea }}\right)^{2}$ were considered. In the case of $Z_{P}$, just before step (2) we remove the Goldstone pole contribution, which, depending directly on the lattice pseudoscalar meson mass, happens to be somewhat different for estimators corresponding to opposite $\theta^{\text {val,sea }}$-values. As for step (4), in the first method ("M1"), after bringing the RC-estimators to a common renormalization scale $\left(\tilde{p}_{\mathrm{M} 1}^{2}=1 / a^{2}\right)$, we remove the remaining $O\left(a^{2} \tilde{p}^{2}\right)$ discretization errors by a linear fit in $\tilde{p}^{2}$. Here the fit range is $1.5 \leq a^{2} \tilde{p}^{2} \leq 2.2$. The second method ("M2") consists in simply taking the value of the RCs estimators at some high momentum point kept fixed in physical units at all $\beta$ 's. Here we choose $\tilde{p}^{2}=12.0 \pm 0.5 \mathrm{GeV}^{2}$. The two approaches yield $\mathrm{RC}$ values differing only by cutoff effects.

In Fig. 1 , for the example of the ensemble $1 \mathrm{p} / \mathrm{m}$ of $\beta=1.95$, we show the Goldstone pole removal and the residual valence quark mass dependence in the analysis of $Z_{P}$ (panel (a)) and the extrapolation to the valence chiral limit for $Z_{q}, Z_{A}, Z_{T}$ and $Z_{V}$ (panel (b)). The fit Ansatz is a linear function of the valence quark polar mass. We checked that results do not change significantly by using more complicated fit functions (involving higher mass powers or $\theta^{\mathrm{val}}$ ). Fig. 2 shows, for $\beta=1.95$ the extrapolation to the sea chiral limit of $Z_{P}, Z_{S}$ (panel (a)) and $Z_{A}, Z_{V}$ (panel (b)). The fit Ansatz is a linear function of $\left(M^{\text {sea }}\right)^{2}$. More elaborated fit functions give compatible results.

For the typical (and important) cases of $Z_{A}$ and $Z_{P}$ we show for $\beta=1.95$ (in Fig. 3) and $\beta=2.1$ (in Fig. 4) the residual dependence on $a^{2} \tilde{p}^{2}$ of RC-estimators (in the case of $Z_{P}$ brought 


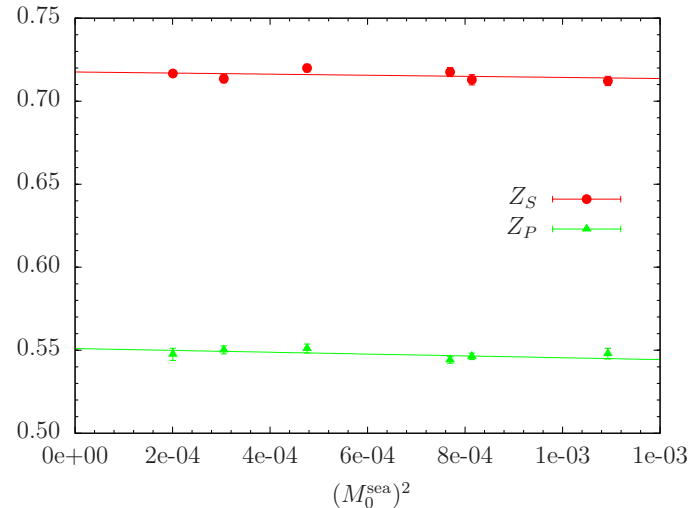

(a)

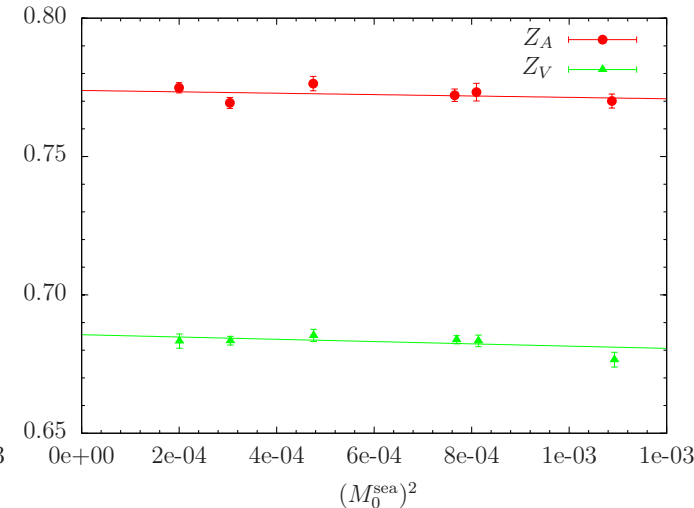

(b)

Figure 2: $\beta=1.95$ : extrapolation to the sea chiral limit for (a) $Z_{P}$ and $Z_{S}$; (b) $Z_{A}$ and $Z_{V}$ (at $\tilde{p}^{2} \sim 11.5 \mathrm{GeV}^{2}$ ).

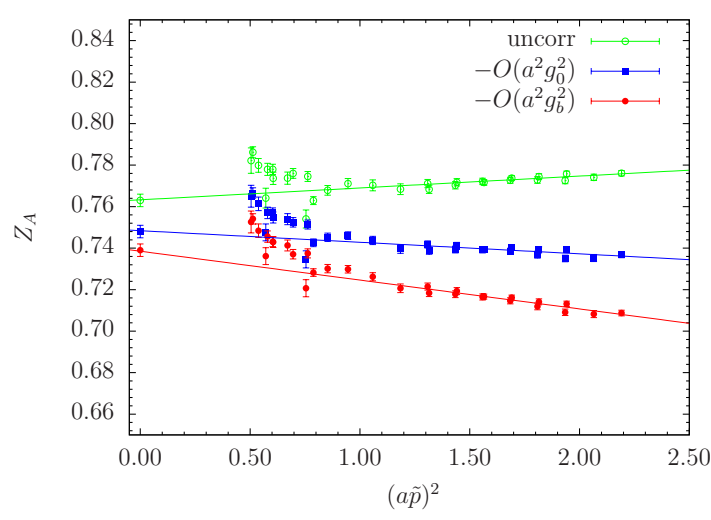

(a)

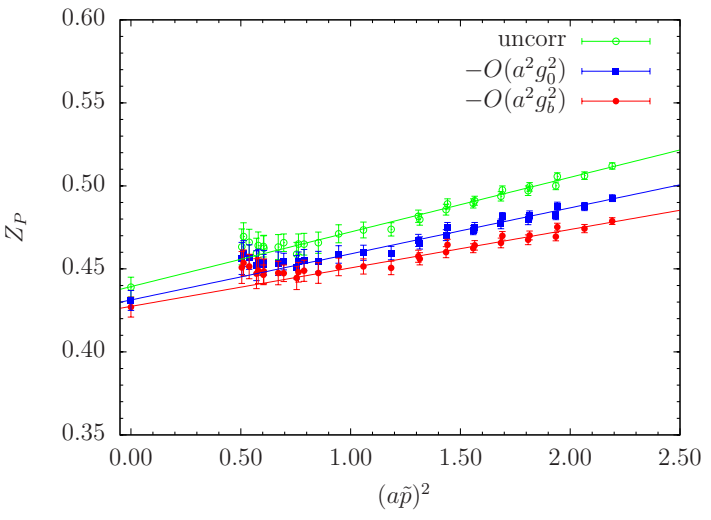

(b)

Figure 3: $\beta=1.95$; dependence of $Z_{A}$ (left panel) and $Z_{P}$ (right panel) on $\left(a^{2} \tilde{p}^{2}\right)$. Uncorrected and one-loop corrected (with two choices of the gauge coupling, bare and plaquette-boosted) RC estimators are shown.

to a common renormalization scale $(1 / a(\beta))$ via three-loop evolution). The nice quality of the linear fit leading to the "M1" RC-values is visible, while results of the "M2" type are obtained from data at $a^{2} \tilde{p}^{2}$ in the range (1.8-2.0) and (1.10-1.23) for $\beta=1.95$ and $\beta=2.1$, respectively. In each plot three different RC-estimators are considered, which differ from each other in the way the (beneficial) subtraction of $\mathrm{O}\left(a^{2} g^{2}\right)$ lattice artefacts of step (1) is carried out.

In Table 2 we gather our preliminary results for the RCs at two values of the gauge coupling, $\beta=1.95$ and $\beta=2.10$. We present results obtained from the two methods described above, namely "M1" and "M2". Perturbative contibutions $\mathrm{O}\left(a^{2} g^{2}\right)$ have been subtracted using the coupling constant estimate $g_{0}^{2}=6 / \beta$. Results for the RCs whose anomalous dimension is non-zero are given at the scale $1 / a$ in the RI/MOM scheme. Following ref. [1], we take $\left.a^{-1}\right|_{\beta=1.95(2.1)}=2.5(3.2) \mathrm{GeV}$.

\section{Acknowledgements}

We thank AuroraScience, Donald Smits center for information technology, University of Groningen, IDRIS, INFN/apeNEXT and Jülich Supercomputing Center for providing the necessary CPU 


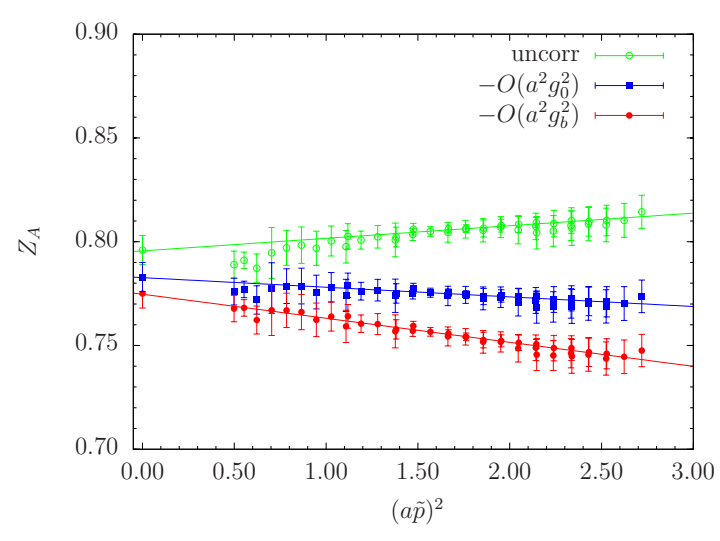

(a)

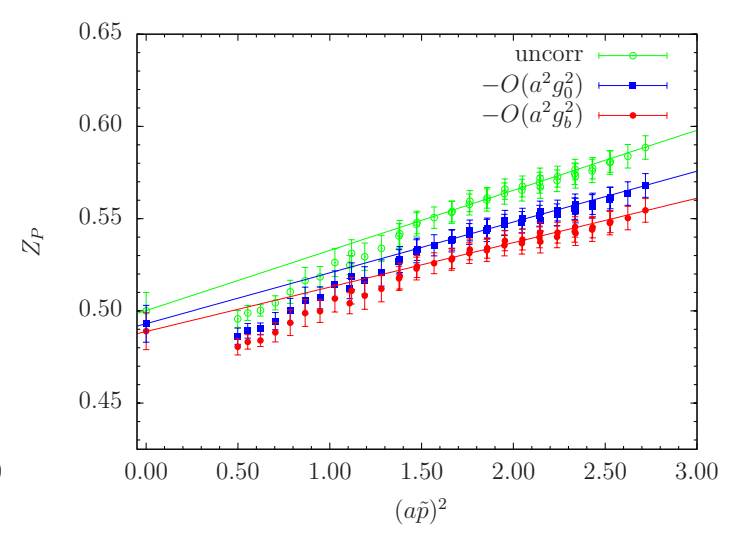

(b)

Figure 4: $\beta=2.10$; dependence of $Z_{A}$ (left panel) and $Z_{P}$ (right panel) on $\left(a^{2} \tilde{p}^{2}\right)$. Uncorrected and one-loop corrected (with two choices of the gauge coupling, bare and plaquette-boosted) RC estimators are shown.

\begin{tabular}{|c|c|c|c|c|c|c|c|}
\hline Method & $Z_{A}$ & $Z_{V}$ & $Z_{P}(1 / a)$ & $Z_{S}(1 / a)$ & $Z_{P} / Z_{S}$ & $Z_{T}(1 / a)$ & $Z_{q}(1 / a)$ \\
\hline \hline \multicolumn{8}{|c|}{$\beta=1.95$} \\
\hline M1 & $0.746(05)$ & $0.614(03)$ & $0.426(06)$ & $0.609(08)$ & $0.700(08)$ & $0.734(04)$ & $0.752(05)$ \\
M2 & $0.738(01)$ & $0.639(02)$ & $0.483(02)$ & $0.684(01)$ & $0.706(03)$ & $0.734(01)$ & $0.769(01)$ \\
\hline \multicolumn{8}{|c|}{$\beta=2.10$} \\
\hline M1 & $0.783(07)$ & $0.683(13)$ & $0.493(10)$ & $0.669(08)$ & $0.737(14)$ & $0.775(11)$ & $0.786(13)$ \\
M2 & $0.777(05)$ & $0.680(05)$ & $0.515(06)$ & $0.696(08)$ & $0.740(08)$ & $0.771(05)$ & $0.794(07)$ \\
\hline
\end{tabular}

Table 2: Our preliminary results for quark bilinear RCs at $\beta=1.95$ and $\beta=2.10$.

time. We acknowledge PRACE Research Infrastructure resource based in Germany at Forschungzentrum Juelich (FZJ) under the project PRA027, ISCRA at CINECA (Italy) under the application HP10A7IBG7 and GENCI Grant 052271 and CC-IN2P3 for partial computer support.

\section{References}

[1] ETMC, R. Baron et al., JHEP 1006 (2010) 111 [1004.5284 [hep-lat]]; ETMC, R. Baron et al. Comput. Phys. Commun. 182 (2011) 299-316 [1005.2042 [hep-lat]].

[2] Y. Iwasaki, Nucl. Phys. B 258 (1985) 141.

[3] R. Frezzotti and G. C. Rossi, JHEP 0408 (2004) 007 [hep-lat/0306014]

[4] R. Frezzotti and G. C. Rossi, Nucl. Phys. Proc. Suppl. 128 (2004) 193 [hep-lat/0311008]

[5] R. Frezzotti and G. C. Rossi, JHEP 0410 (2004) 070 [hep-lat/0407002].

[6] G. Martinelli et al., Nucl. Phys. B 445 (1995) 81 [hep-lat/9411010].

[7] ETMC, M. Constantinou et al. , JHEP 1008 (2010) 068 [1004.1115 [hep-lat]].

[8] ETMC, P. Dimopoulos et al., PoS LATTICE2010 (2010) 235. [1101.1877 [hep-lat]].

[9] R. Frezzotti, G. Martinelli, M. Papinutto, G. C. Rossi, JHEP 0604 (2006) 038. [hep-lat/0503034].

[10] ETMC, N. Carrasco et al., PoS Lattice 2011 (2011) 276 [1111.1262 [hep-lat]].

[11] M. Constantinou et al. JHEP 0910 (2009) 064 [0907.0381 [hep-lat]]. 